\title{
Multiplexed Hybrid ARQ for Energy Efficient Transmissions Under Delay Constraints
}

\author{
Rong Zhang and Lajos Hanzo \\ School of ECS., Univ. of Southampton, SO17 1BJ, UK. \\ Tel: +44-23-80-593 125, Fax: +44-23-80-593045 \\ Email: 1h@ecs.soton.ac.uk, http://www-mobile.ecs.soton.ac.uk
}

\begin{abstract}
A novel Multiplexed Hybrid Automatic Repeat reQuest (M-HARQ) scheme is proposed and compared to the conventional scheme in terms of its energy efficiency, while satisfying specific delay constraints. By employing the proposed scheme, the transmission rate and power can be reduced, while maintaining the maximum energy efficiency and meeting specific delay constraints. Alternatively, the cell-radius distance may be extended by about $10 \%$, which results in a coverage area extension by a factor of 1.21.
\end{abstract}

\section{INTRODUCTION}

Energy-efficient resource utilisation in a given bandwidth while supporting specific Quality of Service (QoS) requirements has recently raised the community's interests in 'green' radio [1]-[3]. Accordingly, the various physical layer elements, such as modulation and coding may be jointly designed with the aid of employing optimisation tools [4] or gametheoretic methods [5] for effectively exploiting the available wireless resources, including the bandwidth and power, subject to given delay constraints. However, Hybrid Automatic Repeat reQuest (HARQ) schemes [6] have not been investigated in terms of their energy efficiency under delay constraints.

Against this background, in this paper we investigate the recently proposed Multiplexed HARQ (M-HARQ) scheme [7] in the context of its achievable energy efficiency, when subject to delay constraints within a given system bandwidth and at a specific source packet arrival rate. The main philosophy of the M-HARQ scheme [7] is that it jointly encodes the current new packet to be transmitted and any packets that are about to be retransmitted. Since the M-HARQ scheme is particularly suitable for low-throughput applications, we focus our attention on the critical cell-edge scenario.

In a nutshell, the contribution of this paper is that we design and investigate the M-HARQ scheme for improving the coverage in the cell-edge scenarios for the sake of maximizing its energy efficiency, while satisfying certain delay constraints.

The rest of the paper is organized as follows. In Section II, we introduce the M-HARQ principle. In Section III, the transmission strategies of both the conventional scheme and of the proposed scheme are discussed in the context of their energy efficiency, when subjected to delay constraints. In Section IV, numerical results are provided for both schemes. Finally, we conclude our discourse in Section V.

Acknowledgments: The financial support of the EPSRC under the auspice of the UK-India Centre of Excellence in Wireless Communications is gratefully acknowledged.

\section{Multiplexed HARQ}

\section{A. The Rationale of $M-H A R Q$}

Being a physical-layer-aware ARQ scheme, HARQ combines the Cyclic Redundancy Check (CRC) encoding function of the link layer with channel coding in the physical layer. In HARQ, the receiver asks for a packet's retransmission using the reverse-direction channel with the aid of a singlebit Negative-ACKnowledgement (NACK) flag, whenever its currently decoded packet is deemed to be erroneous based on the decision of the CRC scheme.

The conventional strategy of transmitting the next new packet only when the successful reception of the current one was confirmed is highly inefficient. However, we may exploit the multiplexing capability inherently provided by channel codes having a channel coding rate $r$ less than unity by superimposing different packets with the aid of their unique, packet-specific interleavers [8]. If the receiver is capable of tolerating a modest amount of additional interference, the next new packet can be simultaneously transmitted with the retransmissions of the previous erroneous packets, as seen in Fig. 1. In other words, the new packets are continuously transmitted, while the erroneous packets are transmitted on a virtual channel, appropriately combined with the new packets.

In general, different packets require different number of retransmissions $L$, depending on the instantaneous channel conditions. We consider the worst-case scenario, where each packet exploited the maximum number of retransmissions. In the worst-case scenario considered and when employing the superposition coding scheme to be introduced shortly, the resultant interference of our M-HARQ arrangement becomes similar to that of the Inter-Symbol-Interference (ISI) effects experienced for transmission over a dispersive channel in the absence of HARQ transmissions. Analogously, our scheme may be interpreted as generating Inter-Packet-Interference (IPI) as seen in Fig. 1.

\section{B. The Construction of M-HARQ}

1) Superposition Encoding: Assume that there are a total of $M$ packets $\mathbf{u}_{m}, m=1, \ldots, M$. Generally speaking, the joint encoding function $F$ of the $m$ th transmission can be represented as $F\left(\mathbf{u}_{a_{1}}, \ldots, \mathbf{u}_{a_{2}}\right)$, where we have:

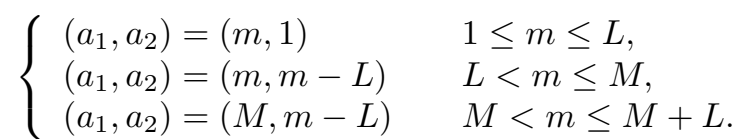




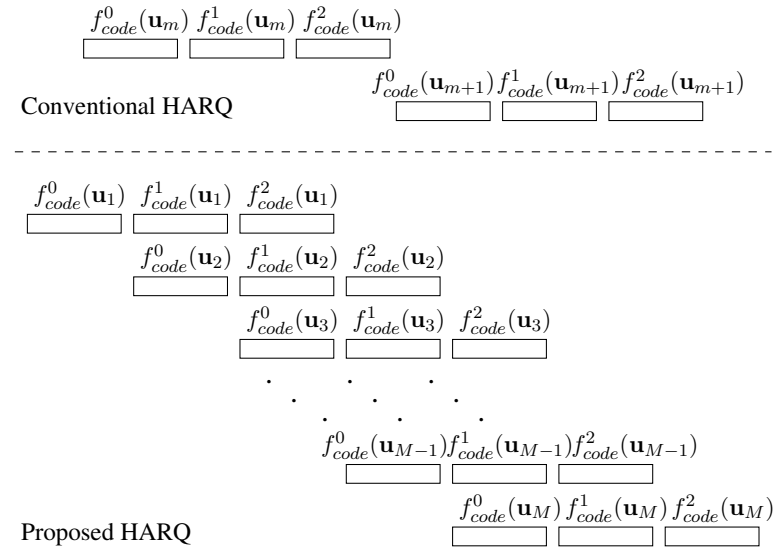

Fig. 1. Classic HARQ and the proposed M-HARQ in conjunction with the number of retransmissions $L=2$ and a total of $M$ transmission packets.

Although in principle specifically designed coding functions may be created, we opt for the powerful superposition coding concept in this paper:

$$
F(\cdot)=\sum_{i=a_{2}}^{a_{1}} \rho_{i} e^{j \theta_{i}} f_{\text {modu }}\left[f_{\text {code }}^{m-i}\left(\mathbf{u}_{i}\right)\right],
$$

where each superimposed packet is referred to as a layer, while $\rho_{i}$ and $\theta_{i} \in[0, \pi)$ denote the layer-specific amplitudeand phase-rotation, respectively. In this paper, an identical amplitude allocation and uniform phase rotations are employed for the individual superimposed layers. The benefit of choosing this particular superposition coding technique is that by opting for this simple linear operation, the specific modulation function $f_{\operatorname{modu}}(\cdot)$ and channel coding function $f_{\text {code }}(\cdot)$ of the individual layers may be retained, where the superscript of the channel coding functions denotes the specific channel code employed for different transmission attempts, which are assumed to be identical in this paper.

2) Iterative Decoding: Our M-HARQ scheme employs iterative Multiple Packets Detection (MPD) and Channel Decoding (DEC) exchanging extrinsic information between these two receiver components. The choice of the DEC algorithm depends on the specific channel code employed, however, a host of MPD schemes may be invoked, including the powerful but high-complexity Maximum Likelihood (ML) detection scheme or we may opt for employing a low-complexity interference cancellation scheme [9] having a linearly increased detection complexity as a function of the number of superimposed packets. The soft-detected packets generated from different transmission attempts may be appropriately combined before soft-decoding or they are individually soft-decoded without the requirements for buffering the previous transmission packets. The latter technique is assumed in this paper.

\section{The performance of $M-H A R Q$}

Let us now evaluate the link layer Packet Error Ratio (PER) performance of our proposed M-HARQ scheme. The top trace of Fig. 2 shows the PER performance of the proposed arrangement against that of the conventional scheme for a total of $L+1=3$ transmissions. In practice, a total of two or three transmissions are sufficient, since the HARQ scheme acts like a 'safety net' in support of the link adaptation procedure, which is capable of preventing most of the potential packet loss events. In our simulations, each source packet of length $N_{s}=256$ bits is channel coded by a rate-1/3 maximum free distance convolutional code and QPSK modulated. A Rayleigh distributed block-fading channel is used under the assumption of perfect channel knowledge at the receiver and the feedback channel conveying the NACK indicator is assumed to be errorfree. We consider the first three transmissions of seen in Fig. 1 , where the three packets experience different interference patterns, ranging from no IPI for the first packet to two interfering packets for the third packet. The top trace of Fig. 2 suggests that all packets experience a near-identical PER performance, when using an iterative receiver, which effectively removes the IPI.

Our proposed scheme is based on the superposition coding approach and hence the resultant composite packet of multiple superimposed layers becomes effectively 'interferencelimited'. Therefore, the per-layer throughput should not be excessive in order to ensure that the decoded PER approaches the single-layer best-case performance. As a result, this requirement discourages the employment of high-order modulation schemes. Furthermore, relatively low-rate channel codes are preferred for the sake of supporting the transmission of multiple superimposed layers at a near-single-layer PER performance. Since the number of retransmissions $L$ is typically low in practice, so is the number of superimposed layers. This makes our scheme particularly suitable for delayconstraint low-rate applications providing cell-edge users with an improved transmission integrity, which will be elaborated more in the following section.

\section{ENERGY EFFICIENCY UNDER DELAY CONSTRAINTS}

\section{A. System Model and Design Metric}

A packet's transmission may be modelled by a queue and a wireless link using the M/G/1 model of [10], which has a Poissonian source packet arrival process having an arrival rate of $\lambda$, a general independently and identically distributed (i.i.d.) packet delivery time $T$ and a single server. We now take into account the constraint imposed on the average delay $D$, which comprises both the packet delivery time $T$ and the queuing delay $D_{q}$. To simplify our system model, we ignore the propagation delay and acknowledgement feedback delay over the wireless channel. Then we have $T+D_{q} \leq D$. Consider an uplink packet transmission from the cell edge using lowrate channel coding and a classic Gray coded square $2^{b}$-ary QAM scheme [11], where $b=2,4$ represents the number of Bits Per Symbol (BPS). More specifically, $b=2$ is employed in the proposed M-HARQ scheme, while $b=2,4$ may be employed in the conventional HARQ scheme. Furthermore, we let $W, R$ and $P$ denote the system's bandwidth, symbol rate and transmission power, respectively.

1) Energy Efficiency Metric: Our objective is to find the best transmission strategy $\mathcal{S}=\{b, R, P\}$, in order to maximize the energy efficiency $\eta$, subject to the average delay constraints $D$ in a given system bandwidth $W$ at a packet arrival rate of 
$\lambda$, which is formulated as:

$$
\max _{\mathcal{S}=\{b, R, P\}} \eta \quad \text { s.t. } \quad T+D_{q} \leq D, \quad R \leq W .
$$

The energy efficiency $\eta$ may be defined as the transmission goodput normalised by the transmission power, which is given by [3], [12]:

$$
\eta=\operatorname{rbR} f(\gamma) / P
$$

where $f(\gamma)$ denotes the Packet Success Ratio (PSR), which is a function of the effective Signal-to-Interference-Noise-Ratio (SINR) denoted by $\gamma=\gamma_{0} G_{c}\left(\gamma_{0}\right)$. The effective SINR is defined as the result of the conventional SINR improved by a certain factor corresponding to the channel coding gain $G_{c}\left(\gamma_{0}\right)$. In other words, the effective SINR $\gamma$ is an up-scaled version of the SINR $\gamma_{0}$, where the amount of noise and interference reduction is controlled by the channel coding gain $G_{c}\left(\gamma_{0}\right)$. For the sake of simplicity, we consider the effective SINR $\gamma$, but without opting for a specific channel code other than taking into account the packet-length extension, which is proportional to the code-rate.

For Gray-coded square $2^{b}$-QAM packets having a length of $N=N_{s} / r$ bits, the PSR is given by [13]:

$$
f(\gamma)=\left[1-p_{P A M}(\gamma)\right]^{2 N / b}-c,
$$

where $p_{P A M}(\gamma)$ is the Symbol Error Ratio (SER) of a $2^{b-1}$ ary PAM scheme [13], while $c=2^{-N}$ is an additive factor employed to ensure that we have $f(\gamma=0)=0$. The received effective SINR $\gamma$ can be written as [5]:

$$
\gamma=\frac{W}{R} \frac{P h}{\sigma^{2}+I}
$$

where $h$ denotes the channel gain, which is the product of the pathloss $h_{p}$, the log-normal shadowing attenuation $h_{s}$ and the frequency flat fading factor $h_{f}$. Furthermore, $I=\sum_{k=1}^{K-1} P_{k} h_{k}$ denotes the total amount of imposed $(K-1)$ co-channel interference and $\sigma^{2}$ is the noise variance after taking into account the channel coding gain.

In our proposed M-HARQ scheme, we exploited the simplifying assumption that each of the three QPSK modulated packets of Fig 2 experienced the same PER performance as if there were no superimposed packets transmitted in parallel. This implies that there is no IPI after the iterative interference cancellation alluded to in the context of the top trace of Fig 2 and the resultant effective SINR of each packet of our MHARQ scheme may also be expressed by Eq (6), while the PSR function of Eq (5) is also applicable to each packet of our M-HARQ scheme. Hence, we may rewrite the energy efficiency of Eq (4) as:

$$
\begin{aligned}
\eta & =\frac{r b R W f(\gamma) h}{R \gamma\left(\sigma^{2}+I\right)} \\
& =W \psi r b f(\gamma) / \gamma \\
& \propto b f(\gamma) / \gamma,
\end{aligned}
$$

where the effective channel gain of $\psi=h /\left(\sigma^{2}+I\right)$ and the system bandwidth $W$ are constant factors and hence can be dropped after normalisation. Eq (9) implies that the energy efficiency $\eta$ becomes a function of the receiver's effective
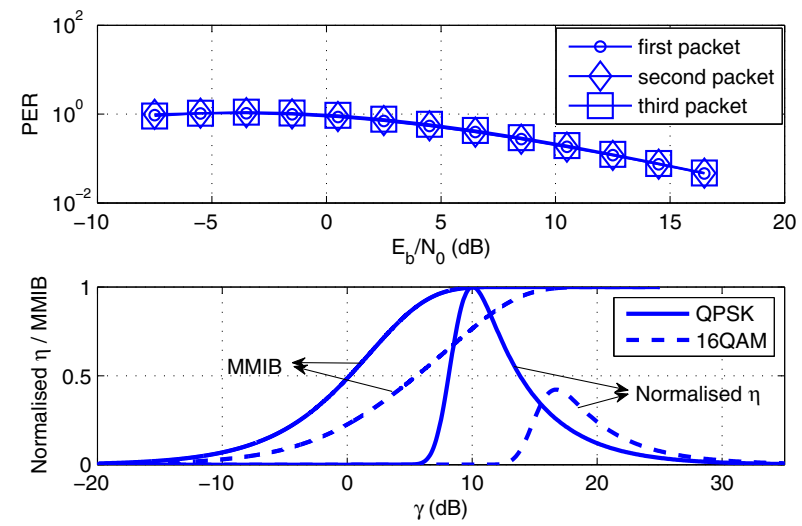

Fig. 2. The PER performance of all three packets with no IPI as well as a single interfering packet and two interfering packets (top); The energy efficiency $\eta$ evaluated from Eq (9) as well as the Mean Mutual Information per Bit (MMIB) $I_{b}$ evaluated from Eq (10) for both QPSK and 16QAM (bottom).

SINR $\gamma$ for a specific number of BPS $b$. It was shown in [3] that the PSR function $f(\gamma)$ is a sigmoidal function, hence Eq (9) indeed has a unique maximum, when we have an effective SINR $\gamma=\gamma^{*}$, which was computed numerically for different values of $b$, as demonstrated in the bottom trace of Fig 2 .

2) Constellation Order: It can be seen in the bottom trace of Fig 2 that QPSK is more energy efficient than 16QAM, where the energy efficiency is normalised by the maximum efficiency value of QPSK. Furthermore, it is also seen in the bottom trace of Fig 2 that the Mean Mutual Information per Bit (MMIB) of QPSK modulation is higher than that of 16QAM, where the MMIB $I_{b}$ may be written as [14]:

$$
I_{b}=\frac{1}{N 2^{b}} \sum_{n=1}^{N} \sum_{i}^{2^{b}} I_{n, i}\left(\gamma_{n}\right),
$$

and $I_{n, i}\left(\gamma_{n}\right)=I_{n, i}\left(x_{n, i}, \mathcal{L}_{x_{n, i}}\right)$ denotes the mutual information of the $i$ th bit of the $n$th symbol $x_{n, i}$ as a function of the $n$th symbol's effective SINR $\gamma_{n}$ of a length- $N$ channel coded packet. For BPSK and QPSK, the Probability Distribution Function (PDF) of the conditional Logarithmic Likelihood Ratio (LLR) $\mathcal{L}_{x_{n, i}}$ is approximately Gaussian [15] and the non-linear function of $\mathrm{Eq}$ (10) can be approximated by a polynomial $J(\gamma)$, as detailed in [16]. By contrast, for 16QAM, the PDF of the conditional LLR can be approximated as a mixture of Gaussian PDFs and the expression of Eq (10) can be written as a weighted sum of $J(\gamma)$, as detailed in [16].

These results suggest the well-recognized natural conclusion that at a given throughput QPSK is more power efficient than 16QAM, since the doubled bandwidth efficiency of 16QAM is achieved at the cost of a typically $6 \mathrm{~dB}$ higher SINR requirement, which leads to a reduced energy efficiency in terms of bits per joule. Hence, when energy efficiency maximisation is the objective, it is always the lowest order modulation should be considered, as long as this choice meets the given delay constraint $D$. However, when the delay constraint is tight, high-order 16QAM transmissions are necessary in order to halve the packet-length at a given throughput or to double the throughput at a given packet-length. 


\section{B. Transmission Strategy for the Conventional HARQ Scheme}

We assume i.i.d. packet error events for transmission over the block fading channel considered in this paper, where the conventional HARQ scheme keeps retransmitting a given packet, until its successful reception is declared. For the M/G/1 queue, the average delay constraint may be quantified by the Pollaczek-Khintchine formula [10]:

$$
E(T)+\lambda\left[E(T)^{2}+\sigma_{T}^{2}\right] /[2-2 \lambda E(T)] \leq D,
$$

where $E(T)$ and $\sigma_{T}^{2}$ denote the Average Successful Packet Delivery Time (ASPDT) and its variance, respectively. Since i.i.d. packet loss events are assumed, the probability of the $l$ th transmission (i.e. the $(l-1)$ st retransmission) of a packet succeeding is given by $\operatorname{Pr}(l)=f(\gamma)[1-f(\gamma)]^{l-1}$. Then, the ASPDT and its variance are given by [5]:

$$
\begin{aligned}
E(T) & =\tau / f(\gamma), \\
\sigma_{T}^{2} & =[1-f(\gamma)] \tau^{2} / f(\gamma)^{2},
\end{aligned}
$$

where the packet duration is $\tau=N / b R$. By substituting Eq (12) and Eq (13) into Eq (11), the resultant delay constraint may be formulated in conjunction with the PSR $f(\gamma)$ as:

$$
N / b R D+\lambda N / b R-\lambda N^{2} / 2 b^{2} R^{2} D \leq f(\gamma) .
$$

Let the function $g(b, R, \lambda, D)$ denote the Left Hand Side (LHS) of Eq (14). Given these preliminaries, we now find the best transmission strategy $\mathcal{S}$ as detailed below:

For a given source packet arrival rate $\lambda$ and delay constraint $D$, the lowest number of BPS $b^{*}$ still capable of meeting the delay constraint of Eq (14) is obtained, when we have a $g\left(b^{*}, R, \lambda, D\right)$ value at the LHS of Eq (14) is at its minimum, while $f(\gamma)$ at the Right Hand Side (RHS) of Eq (14) is at its maximum. This means that upon transmitting at the maximum possible symbol rate of $R=W$ and aiming for a near-unity PSR of $f(\gamma) \rightarrow 1$, then we have $g\left(b^{*}, W, \lambda, D\right)<1$.

For the sake of achieving the maximum attainable energy efficiency $\eta^{*}$, we have to maintain the optimum effective SINR $\gamma^{*}$, which implies that at a chosen $b^{*}$ and given $\lambda$ as well as $D$, we have to transmit at a symbol rate of $R^{*}$ to ensure that a PSR of $f\left(\gamma^{*}\right)$ is maintained, which means that we have $g\left(b^{*}, R^{*}, \lambda, D\right)=f\left(\gamma^{*}\right)$. As a result, the maximum energy efficiency $\eta^{*}$ is achieved, as long as we have $R^{*} \leq W$ and the associated transmit power obeys $P^{*}=\gamma^{*} R^{*} / \psi W$.

However, when we have an excessive symbol rate of $R^{*}>$ $W$, achieving this energy efficiency maximum becomes unrealistic, since we have to obey the maximum symbol rate constraint of $W$ and hence $g\left(b^{*}, W, \lambda, D\right)>f\left(\gamma^{*}\right)$. In this case, the resultant equivalent SINR becomes $f^{-1}\left[g\left(b^{*}, W, \lambda, D\right)\right]>$ $\gamma^{*}$ and the associated transmit power is given by $P=$ $f^{-1}\left[g\left(b^{*}, W, \lambda, D\right)\right] / \psi$.

This implies that the maximum energy efficiency $\eta^{*}$ can be always maintained by choosing the appropriate combinations of symbol rate $R^{*}$ and power $P^{*}$, provided that $R^{*} \leq W$ is satisfied. After the symbol rate reaches its maximum $W$, when the delay constraint becomes tighter, the transmission power $P$ has to be further increased to ensure that we maintain an effective SINR of $\gamma>\gamma^{*}$, which reduces the achievable energy efficiency.

\section{Transmission Strategy for the Proposed M-HARQ Scheme}

For the proposed M-HARQ scheme, the transmission strategy $\mathcal{S}$ becomes slightly different. In M-HARQ, the retransmission of the current erroneous packet and the transmission of the next new packet are superimposed. The benefit of this is that it requires no 'channel-reservation' for the retransmission of the current packet. We assume that the IPI may be mitigated at the receiver without any performance degradation with the aid of interference cancellation, as long as the number of superimposed packets is not excessive. Indeed, this is typically the case in practice, where a total of two or three transmission attempts of a packet are sufficient. Hence, the ASPDT $E(T)$ is exactly the same as the packet duration $\tau$. Then the delay constraint of Eq (11) becomes equivalent to $g(b, R, \lambda, D) \leq 1$. The corresponding transmission strategy may be formulated as detailed below:

Firstly, we find the lowest number of BPS $b^{*}$, which can be transmitted, while ensuring that the delay constraint $D$ is met at the symbol rate of $R=W$ for a given source packet arrival rate $\lambda$. At this value of $b^{*}$, we may find the appropriate symbol rate $R^{*}$, at which $g\left(b^{*}, R^{*}, \lambda, D\right)=1$ holds. We then have to transmit at a power of $P^{*}=\gamma^{*} R^{*} / \psi W$ in order to achieve the maximum energy efficiency $\eta^{*}$. By employing the proposed scheme, the maximum energy efficiency $\eta^{*}$ can be maintained by choosing the appropriate combinations of $R^{*}$ and $P^{*}$, given that $R^{*} \leq W$. By contrast, when an excessive symbol rate of $R^{*}>W$ would be required to maintain the delay constraint of $D$, then using a higher-order constellation size becomes necessary to either halve the packet-length or to double the packet's payload.

\section{NumERiCAL RESUlts}

In this section we contrast the conventional and proposed scheme in terms of their energy efficiency, while satisfying a given delay constraint. Explicitly, the information packet size is assumed to be $N_{i}=80$ bits, protected by a channel code having a rate of $r=1 / 3$, which results in a channel coded packet size of $N=240$ bits. We assign a system bandwidth of $W=5 \mathrm{MHz}$ and employ the normalised delay constraints of $D_{n}=D W=\left[10^{2}, \ldots, 10^{4}\right]$. The normalised packet arrival rates used are $\lambda_{n}=\lambda / W=[0.001,0.0001]$. Finally, the normalised transmission rate is defined as $R_{n}=b R / W$. The transmission power $P$ and energy efficiency $\eta$ are normalised by $\psi$ and $\psi W r$, resulting $P_{n}$ and $\eta_{n}$, respectively.

1) Transmission Rate and Power: Let us first investigate the achievable normalised transmission rate and power in Fig 3. The switch point indicated by a circle in the figure represents a change of constellation size between $b=2$ and $b=4$. Since only QPSK was implemented in our proposed M-HARQ scheme, the 16QAM aided M-HARQ scheme is referred to as 'Not Applicable (NA)', which is hence not used for comparison. Fig 3 demonstrates that both schemes would have to choose the modulation order under the given delay constraint at the same switch point. As the delay constraint becomes tighter, an increased transmission rate and power is required for satisfying it. However, the proposed scheme requires a consistently lower transmission rate and power than 

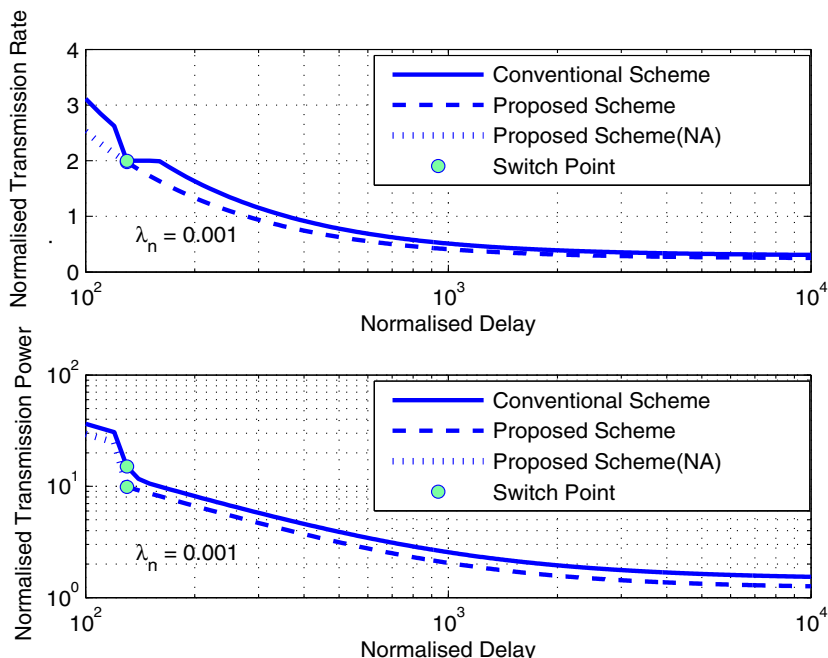

Fig. 3. Normalised transmission rate (top) and power (bottom) under normalised delay constraints.

the conventional scheme in order to meet the delay constraint stipulated, while maintaining the maximum energy efficiency, as illustrated in the top trace of Fig 4.

2) Energy Efficiency and Coverage Gain: The top trace of Fig 4 compares the energy efficiency of both schemes, where it is observed that the proposed scheme maintains at least the same or higher energy efficiency than that of the conventional scheme. In particular, observe in the top trace of Fig 3 that when $b=2$ is considered, for the conventional scheme a PSR of $f(\gamma)>f\left(\gamma^{*}\right)$ is required, when transmitting at a symbol rate of $R=W$ under a tight delay constraint, which results in a reduced energy efficiency $\eta$, as seen in the top trace of Fig 4, since the required increase in these PSR $f(\gamma)$ is achieved by increasing the effective SINR $\gamma$. On the other hand, when $b=2$ is considered, our proposed scheme achieves the maximum attainable energy efficiency for symbol rates of $R \leq W$ for all delay constraints considered.

The benefit of requiring a consistently reduced transmission power for the proposed scheme may also be translated into having an enlarged coverage distance. The required transmission powers of the proposed and conventional schemes obey $P_{p} / P_{c}=\left(d_{p} / d_{c}\right)^{-\alpha}$, where the subscript $(\cdot)_{p}$ and $(\cdot)_{c}$ denote the proposed scheme and the conventional scheme, respectively, while $\alpha=2$ is the pathloss exponent. The achievable coverage gain is illustrated in the bottom trace of Fig 4, which shows that our proposed scheme achieves an at least $10 \%$ higher cell-radius distance than that of the conventional scheme.

\section{CONCLUSION}

In this paper, we investigated the proposed M-HARQ scheme, which outperformed the benchmaker conventional HARQ scheme in challenging cell-edge scenarios in the context of its energy efficiency. It is capable of transmitting at a reduced power, while meeting the delay constraints and is also capable of increasing the cell-radius distance by about $10 \%$ and hence the coverage area by about $20 \%$.
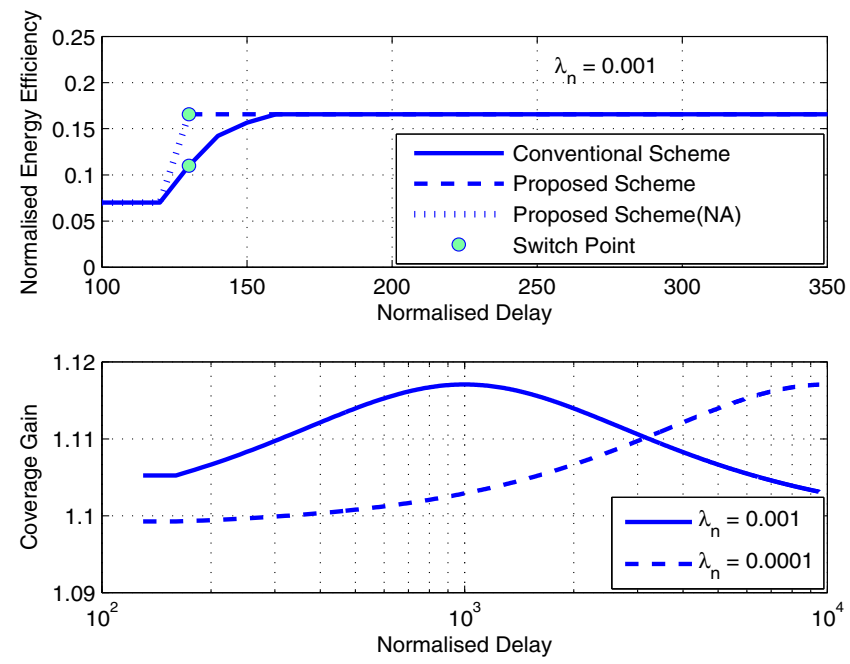

Fig. 4. Normalised transmission energy efficiency (top) and coverage gain (bottom) under normalised delay constraints

\section{REFERENCES}

[1] "CORE-5 Research Area: Green Radio," Virtual Centre of Excellence in Mobile and Personal Communications (MVCE), 2009, http://www.mobilevce.com/infosheets/GreenRadio.pdf.

[2] E. Uysal-Biyikoglu and A. El Gamal, "Energy-efficient packet transmission over a multiaccess channel," in IEEE Proceedings of ISIT '02, Lausanne, Switzerland, 2002, p. 153.

[3] F. Meshkati, H. Poor, and S. Schwartz, "Energy-efficient resource allocation in wireless networks," IEEE Signal Processing Magazine, vol. 24, pp. 58-68, May 2007.

[4] F. Kelly, A. Maulloo, and D. Tan, "Rate control in communication networks: shadow prices, proportional fairness and stability," Journal of the Operational Research Society, vol. 49, pp. 237-252, Mar. 1998.

[5] F. Meshkati, A. Goldsmith, H. Poor, and S. Schwartz, "A game-theoretic approach to energy-efficient modulation in CDMA networks with delay QoS constraints," IEEE Journal of Selected Areas in Communications, vol. 25, pp. 1069-1078, Aug. 2007.

[6] S. Lin and D. J.Costello, Error Control Coding: Fundamentals and Applications., 2nd ed. New York: Prentice-Hall, Inc., 2005.

[7] R. Zhang and L. Hanzo, "Superposition-coding aided multiplexed hybrid ARQ scheme for improved end-to-end transmission efficiency," IEEE Transactions on Vehicular Technology. [Online]. Available: http://eprints.ecs.soton.ac.uk/17230/

[8] _ - "Three design aspects of multicarrier interleave division multiple access," IEEE Transactions on Vehicular Technology, vol. 57, pp. 36073617, Nov. 2008.

[9] X. D. Wang and H. V. Poor, "Iterative (turbo) soft interference cancellation and decoding for coded CDMA," IEEE Transactions on Communications, vol. 47, pp. 1046-1061, July 1999.

[10] W. R. Stevens, TCP/IP Illustrated, Volume I: The Protocols. MA: Addison-Wesley, 1994.

[11] L. Hanzo, S. X. Ng, T. Keller, and W. T. Webb, Quadrature Amplitude Modulation: From Basics to Adaptive Trellis-Coded, TurboEqualised and Space-Time Coded OFDM, CDMA and MC-CDMA Systems. Wiley-IEEE Press, 2004.

[12] C. Saraydar, N. Mandayam, and D. Goodman, "Efficient power control via pricing in wireless data networks," IEEE Transactions on Communications, vol. 50, pp. 291-303, Feb. 2002.

[13] J.G.Proakis, Digital Communications. New York: McGrawHill, 2001.

[14] G. Caire, G. Taricco, and E. Biglieri, "Bit-interleaved coded modulation," IEEE Transactions on Information Theory, vol. 44, pp. 927-946, May 1998

[15] S. ten Brink, "Convergence behavior of iteratively decoded parallel concatenated codes," IEEE Transactions on Communications, vol. 49, pp. 1727-1737, Oct. 2001.

[16] "IEEE 802.16m Evaluation Methodology Document," IEEE 802.16 Broadband Wireless Access Working Group, 2008, http://ieee802.org/16. 